both bNAbs. This suggests that a more-sensitive test than that used by the authors will be needed to assess whether people are likely to respond to a particular bNAb combination.

In the war against HIV, there has been some controversy regarding the use of bNAbs as a treatment approach. Antibodies have been thought of as providing defences equivalent to a naval blockade, stopping HIV from initially infecting or preventing the infection from spreading. But once this barrier is breached and infection takes hold, it is assumed that $\mathrm{T}$ cells are the front line of immune defence against infected cells, with antibodies relegated to bystander status. However, models of infection with a simian-human chimaeric virus called SHIV in primates suggest that antibodies might have an active role in tackling infected cells.

In these primate models, human antibodies become widely distributed in tissues ${ }^{16}$, and could be acting in a manner equivalent to minesweepers, by limiting the spread of infection. Not only are such antibodies distributed efficiently to tissues far from the site of antibody administration, but they can also help to destroy infected cells, at least in the early stages of an infection, and to prevent the establishment of the viral reservoir ${ }^{16,17}$. Once viral infection is established, the introduction of bNAbs that target Env can suppress the virus to undetectable levels in the bloodstream, as long as the antibodies persist there ${ }^{18,19}$. The role of these antibodies in tissues is not fully understood. When the antibody levels decay, viral rebound occurs and immune defences are mediated mainly by $\mathrm{T}$ cells ${ }^{20}$.

Given the results in primates, is there any evidence that bNAbs can tackle the viral reservoir in human HIV infection? Mendoza and colleagues used an in vitro quantitative virusoutgrowth test to assess the viral-reservoir size. They found no significant differences in reservoir size before and after bNAb treatment. However, longer and more-effective bNAb treatment and larger study groups might be needed to establish definitively whether bNAbs affect the viral reservoir.

As strategies are sought to target the reservoir, it is encouraging to know that bNAbs might provide a useful weapon in the arsenal of viral-control tools. Moreover, if it turns out that a cocktail of bNAbs could act as a potential temporary replacement for ART, this type of 'drug holiday' might give people time to recover from ART-induced toxicity.
Nancy L. Haigwood is in the Division of Pathobiology \& Immunology, Oregon National Primate Research Center, Oregon Health \& Science University, Beaverton, Oregon 97006, USA.

e-mail:haigwoon@ohsu.edu

1. Paula, A. A., Falcão, M. C. N. \& Pacheco, A. G. AIDS Res. Ther. 10, 32 (2013)

2. Mendoza, P. et al. Nature 561, 479-484 (2018)

3. Bar-On, Y. et al. Nature Med. https://doi. org/10.1038/s41591-018-0186-4 (2018)

4. Sengupta, S. \& Siliciano R. F. Immunity 48, 872-895 (2018).

5. Boritz, E. A et al. Cell 166, 1004-1015 (2016)

6. Pegu, A., Hessell, A. J., Mascola, J. R. \& Haigwood, N. L. Immunol. Rev. 275, 296-312 (2017).

7. Caskey, M. et al. Nature 522, 487-491 (2015).

8. Bar, K. J. et al. N. Engl. J. Med. 375, 2037-2050 (2016).

9. Scheid, J. F. et al. Nature 535, 556-560 (2016).

10.Ledgerwood, J. E. et al. Clin. Exp. Immunol. 182, 289-301 (2015).

11. Mayer, K. H. et al. PLoS Med. 14, e1002435 (2017). 12.Scheid, J. F. et al. Science 333, 1633-1637 (2011).

13.Zhou, T. et al. Science 329, 811-817 (2010).

14. Rothenberger, M. K. et al. Proc. Natl Acad. Sci. USA 112, E1126-E1134 (2015).

15. Kearney, M. F. et al. PLoS Pathog. 10, e1004010 (2014).

16. Hessell, A. J. et al. Nature Med. 22, 362-368 (2016)

17. Liu, J. et al. Science 353, 1045-1049 (2016).

18. Shingai, M. et al. Nature 503, 277-280 (2013).

19.Barouch, D. H. et al. Nature 503, 224-228 (2013).

20.Nishimura, Y. et al. Nature 543, 559-563 (2017).

\title{
Complex motion guided without external control
}

Mechanical structures have been made that exhibit self-guided, multi-step sequences of shape changes in response to an applied force. Such structures could have applications in flexible electronics and soft robotics. SEE LETTER P.512

\section{LARRY L. HOWELL}

$\mathrm{I}$ $\mathrm{t}$ is reasonable to expect that to guide a mechanical structure through a complex, predetermined series of shape changes, electronic controls would be needed. In many cases, motors move the structure, sensors detect position and electronic devices called microcontrollers execute algorithms to provide external control. Achieving complex motion using only the structure's geometry might seem unrealistic, but, if possible, it would have many advantages. In particular, it would simplify the manufacture of mechanical structures and enable them to be readily produced in various sizes. On page 512, Coulais et al. ${ }^{1}$ report an approach for designing materials that follow a prescribed motion and that then reconfigure at specified points to enter another phase of motion. This movement is guided only by the material's geometry, which eliminates the need for external sensing and control.
Engineered structures known as mechanical metamaterials are designed to behave in ways not common in nature ${ }^{2-4}$. They are usually made by combining building blocks (unit cells) into patterns, to produce a combined structure that can behave in ways not possible using a solid material. Advances in materials, manufacturing, computational capabilities and mechanical-design approaches are enabling designs that were not previously possible, such as the mechanical metamaterials described by Coulais and colleagues.

The authors used thin, flexible parts, strategically combined with thick, stiff parts, to achieve the desired structural motion. They refer to the movement of these parts as soft deformation, because it is caused by the bending of the flexible parts, rather than by mechanical hinges. This type of motion is not new, of course, because it can be seen extensively in nature. Consider, for example, the movement of an elephant's trunk, your beating heart or a butterfly's flapping wings - in each case, motion results from the bending of flexible parts.

Designs in which a combination of flexible and stiff components enables a specified motion are often referred to as compliant mechanisms ${ }^{5}$. The use of these mechanisms in engineered materials offers several advantages over conventional design methods, such as the ability to achieve complex motion from a single part rather than from an assembly of pieces connected by hinges. Coulais et al. used compliant mechanisms to realize surprisingly sophisticated multi-step motions (Fig. 1). One clever aspect of their approach is the use of parts that have different degrees of flexibility, so that, in response to an external force, some parts deform before others do.

Coulais and colleagues also used what they call self-contact, whereby a stiff part is intentionally brought into contact with another stiff part to prevent further motion between the two. This 'contact-aided' mechanism ${ }^{6,7}$ is similar to a door-stop that prevents a door from colliding with a wall. In the authors' approach, bending of the most flexible part results in selfcontact, which ends the first step of a multi-step pathway (Fig. 1a). The most flexible part then becomes inactive, and the second step begins (Fig. 1b). The next-most flexible part becomes the main source of deformation, and this part bends until the next occurrence of self-contact.

The authors produced unit cells from these flexible and stiff components, and combined the unit cells into patterns to achieve the desired motions. In one demonstration, they considered unit cells consisting of a cross-shaped arrangement of five equally sized squares, which were 


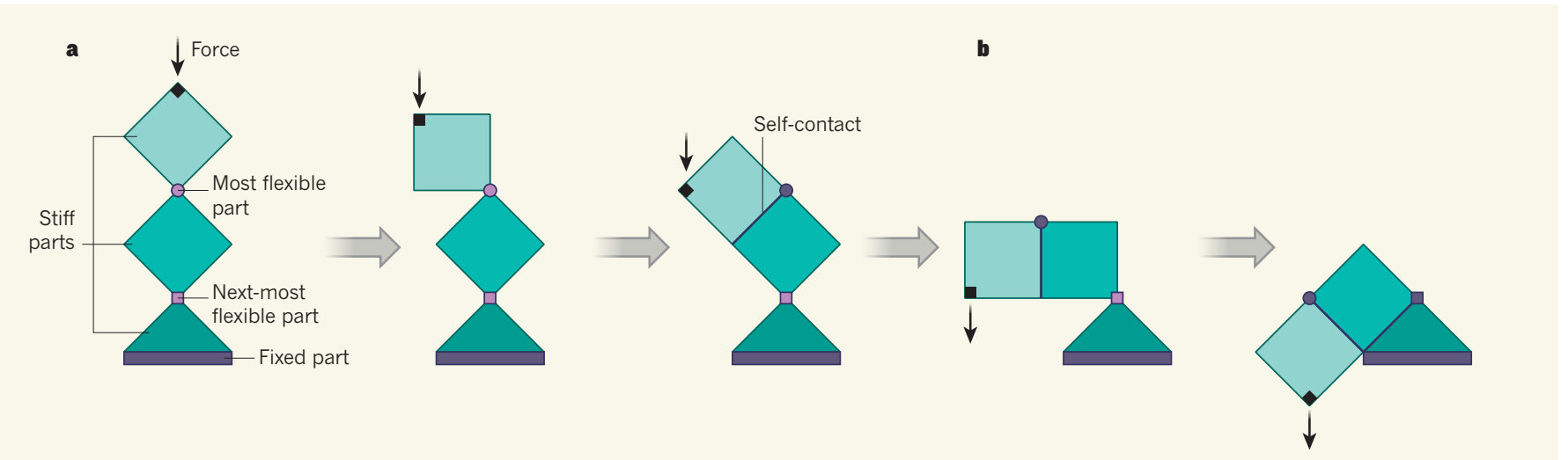

Figure 1 | Self-guided, multi-step motions of a mechanical structure. Coulais et al. ${ }^{1}$ demonstrate an approach for designing materials that follow a multi-step pathway consisting of a series of shape changes, without needing external control. The materials are composed of building blocks (unit cells), and a subset of a unit cell is shown here. a, The subset consists of stiff parts that are connected by flexible parts, and a part that is fixed. The flexible parts have different degrees of flexibility. The authors apply an external force to the structure (at the point marked by the black square), which causes the most flexible part to bend. This bending moves one of the stiff parts until it connects with another stiff part - a process known as self-contact. The subset reconfigures such that the most flexible part becomes inactive and the connected parts act as a single stiff part. The first step of the multi-step pathway is complete. $\mathbf{b}$, The next-most flexible part then bends until another self-contact occurs, which prevents further motion. The desired configuration is reached, and the second step of the pathway is concluded. connected by flexible parts (see Fig. 1a of the paper ${ }^{1}$ ). In the first step of the multi-step pathway, each unit cell rotated to produce a structure of differently sized squares. In the second step, each of these squares rotated to fill an assigned space.

Coulais and colleagues illustrated the concepts associated with their approach using only a few specific examples, but their work provides guidelines that will enable the design of other shape-changing metamaterials. The authors' unit cells can be configured in many different ways, resulting in different motions. For example, two structures that have nearly identical topologies can have contrasting pathways (see Fig. 1a,b of the paper ${ }^{1}$ ). Even more configurations are made possible by producing different arrays of the unit cells, or by using the principles discussed in the paper to make other unit cells and arrays.

The approach does have some limitations. For instance, the flexible parts are relatively small and undergo $90^{\circ}$ of rotation, and few materials can withstand such large strains without breaking. Even materials that do not break might still become permanently deformed, making the process irreversible - and therefore unsuitable for many applications. The authors used 10-millimetrethick silicon rubber for the flexible parts in their experiments. Although the use of this material is valuable for verifying and demonstrating the approach, issues will probably arise when smaller parts are required, because the material choice will be limited.

Coulais and colleagues' results open up many avenues for future research. A natural next step would be to see what other multi-step motions can be realized using the approach. Researchers could also study how the designs vibrate when they are shaken, to see whether they still undergo the intended movements, or whether they follow a different pathway.
In addition, it would be useful to explore whether switch-type mechanisms ${ }^{8,9}$ can be used to snap the structures into desired configurations, replacing the self-contact function and perhaps eliminating the need for an external force to keep the structures in key intermediate positions. Finally, researchers could investigate how the principles described in the current work could be used to produce sophisticated motions beyond multi-step pathways, and how the approach might be applied to the micrometre and nanometre scales..

Larry L. Howell is in the Department of Mechanical Engineering, Brigham Young University, Provo, Utah 84602, USA.

e-mail:lhowell@byu.edu
1. Coulais, C., Sabbadini, A., Vink, F. \& van Hecke, M. Nature 561, 512-515 (2018).

2. Overvelde, J. T. B., Weaver, J. C., Hoberman, C. \& Bertoldi, K. Nature 541, 347-352 (2017).

3. Reis, P. M., Jaeger, H. M. \& van Hecke, M. Extreme Mech. Lett. 5, 25-29 (2015).

4. Haghpanah, B., Salari-Sharif, L., Pourrajab, P., Hopkins, J. \& Valdevit, L. Adv. Mater. 28, 7915-7920 (2016).

5. Howell, L. L., Magleby, S. P. \& Olsen, B. M. Handbook of Compliant Mechanisms (Wiley, 2013).

6. Hopkins, J. Shaw, L., Dotson, M., Chizari, S. \& Song, Y. Bull. Am. Phys. Soc. abstr. F17.00004 (2018).

7. Mankame, N. D. \& Ananthasuresh, G. K. Comput. Struct. 82, 1267-1290 (2004).

8. Silverberg, J. L. et al. Nature Mater. 14, 389-393 (2015).

9. Waitukaitis, S., Menaut, R., Chen, B. G. \& van Hecke, M. Phys. Rev. Lett. 114, 055503 (2015).

\title{
Designer proteins activate fluorescence
}

\begin{abstract}
A computational method has been devised that allows a structural motif found in proteins, known as a $\beta$-barrel, to be designed to bind specifically to any small molecule, opening the door to biotechnological applications. SEE ARTICLE P.485
\end{abstract}

\section{ROBERTO A. CHICA}

$\mathrm{P}$ roteins are the molecular machines of life: they carry out the complex molecular processes required by cells with unrivalled accuracy and efficiency. Many of these processes depend on proteins having the ability to bind specifically to a given small molecule. If we could make proteins from scratch to bind any desired target molecule, it would open the door to a wide range of biotechnological applications that are not currently possible using natural proteins. On page 485 , Dou et al. ${ }^{1}$ describe a computational method for designing proteins tailored to bind a small molecule of interest, and use it to make 'fluorescence-activating' proteins - biotechnological tools that have potential applications in biomedical research.

The functions of proteins are dictated by 